PROCEEDINGS OF THE

AMERICAN MATHEMATICAL SOCIETY

Volume 125, Number 1, January 1997, Pages 229-234

S 0002-9939(97)03649-6

\title{
DENSITIES WITH THE MEAN VALUE PROPERTY FOR HARMONIC FUNCTIONS IN A LIPSCHITZ DOMAIN
}

\author{
HIROAKI AIKAWA
}

(Communicated by J. Marshall Ash)

\begin{abstract}
Let $D$ be a bounded domain in $\mathbb{R}^{n}, n \geq 2$, and let $x_{0} \in D$. We consider positive functions $w$ on $D$ such that $h\left(x_{0}\right)=\left(\int_{D} w d x\right)^{-1} \int_{D} h w d x$ for all bounded harmonic functions $h$ on $D$. We determine Lipschitz domains $D$ having such $w$ with $\inf _{D} w>0$.
\end{abstract}

\section{INTRODUCTION}

Let $D$ be a bounded domain in $\mathbb{R}^{n}, n \geq 2$, and let $x_{0} \in D$. By $\mathcal{H}_{b}$ we denote the set of all bounded harmonic functions on $D$. Hansen and Netuka [3] considered positive functions $w$ on $D$ such that

$$
h\left(x_{0}\right)=\frac{1}{\int_{D} w d x} \int_{D} h w d x \quad \text { for } h \in \mathcal{H}_{b} .
$$

They proved the following theorem.

Theorem A. (i) There exists a domain $D$ which fails to have $w$ satisfying (1) and $\inf _{D} w>0$.

(ii) For every bounded domain $D$ there is positive $w \in C^{\infty}(D)$ satisfying (1) and $\lim _{x \rightarrow \partial D} w(x)=0$.

(iii) For a $C^{1+\varepsilon}$-domain (or a Liapunov-Dini domain (cf. [5])) there is $w \in$ $C^{\infty}(D)$ satisfying (1) and $\inf _{D} w>0$.

We say that a bounded domain $D$ is $k$-Lipschitz if $D$ and $\partial D$ are given locally by a Lipschitz function whose Lipschitz constant is at most $k$. If $D$ is $k$-Lipschitz for some $k>0$, then we say that $D$ is a Lipschitz domain. In this note we consider the same problem for a Lipschitz domain. Our result is the following.

Theorem. (i) There exists a $1 / \sqrt{n-1}$-Lipschitz domain which fails to have $w$ satisfying (1) and $\inf _{D} w>0$.

(ii) If $0<k<1 / \sqrt{n-1}$, then for every $k$-Lipschitz domain $D$ there is $w \in$ $C^{\infty}(D)$ satisfying (1) and $\inf _{D} w>0$.

The author would like to thank Professor Hansen and Professor Netuka for showing him a preprint of [3]. Professor Hansen also pointed out an error in the first version of the paper and showed that the difficulty could be avoided by a general

Received by the editors July 13, 1995 and, in revised form, August 1, 1995.

1991 Mathematics Subject Classification. Primary 31A05, 31B05.

Key words and phrases. Harmonic functions, mean value property, gradient of harmonic function.

(C) 1997 American Mathematical Society 
lemma which A. Ancona communicated to him. The author would like to express his sincere gratitude to Professor Hansen.

\section{Proof of Theorem (i)}

Let $T_{\psi}=\left\{x=\left(x_{1}, \ldots, x_{n}\right): x_{n}>|x| \cos \psi\right\}$. This is an infinite cone with vertex at 0 and aperture $\psi$. By $u_{\psi}$ (resp. $v_{\psi}$ ) we denote the Martin kernel for $T_{\psi}$ with pole at $\infty$ (resp. at 0 ). It is known that $u_{\psi}$ (resp. $v_{\psi}$ ) is of homogeneous of degree $\alpha=\alpha_{n}(\psi)$ (resp. of degree $2-n-\alpha$ ). The constant $\alpha_{n}(\psi)$ is called the maximal order of barriers. It is not so difficult to see that $\alpha_{n}$ is strictly decreasing; $\alpha_{n}(\pi / 2)=1 ; \alpha_{n}(\psi)=2$ for $\cos \psi=1 / \sqrt{n} ; \lim _{\psi \rightarrow 0} \alpha_{n}(\psi)=\infty ; \lim _{\psi \rightarrow \pi} \alpha_{n}(\psi)=0$ (for $n \geq 3$ ); $\alpha_{2}(\psi)=\pi /(2 \psi)$; and $\alpha_{4}(\psi)=\pi / \psi-1$. For the above result see [1] and the references therein. By $B(x, r)$ we denote the open ball with center at $x$ and radius $r$.

Proof of Theorem (i). In this proof we let $\cos \psi=1 / \sqrt{n}$. Then $\tan \psi=\sqrt{n-1}$ and

$$
T_{\psi}=\left\{x=\left(x^{\prime}, x_{n}\right): x_{n}>\frac{1}{\sqrt{n-1}}\left|x^{\prime}\right|\right\} .
$$

Hence it is easy to construct a $1 / \sqrt{n-1}$-Lipschitz domain $U_{\psi}$ such that $U_{\psi} \cap$ $B(0,1)=T_{\psi} \cap B(0,1)$. Let $x_{0} \in U_{\psi}$ be a fixed point and let $K_{0}$ be the Martin kernel for $U_{\psi}$ with pole at 0 with reference point at $x_{0}$. We observe that $K_{0}$ is comparable with $v_{\psi}$ near the origin. Since $\alpha_{n}(\psi)=2$, it follows that $v_{\psi}$ is of homogeneous of degree $-n$ and hence $K_{0}$ is not integrable over $U_{\psi}$. Put

$$
K_{j}=\frac{\omega\left(\cdot, B(0,1 / j) \cap \partial U_{\psi}\right)}{\omega\left(x_{0}, B(0,1 / j) \cap \partial U_{\psi}\right)},
$$

where $\omega$ denotes the harmonic measure for $U_{\psi}$. Then $K_{j} \in \mathcal{H}_{b}\left(U_{\psi}\right), K_{j}\left(x_{0}\right)=1$ and $K_{j} \rightarrow K_{0}$ locally uniformly on $U_{\psi}$. By Fatou's lemma

$$
\liminf _{j \rightarrow \infty} \int_{U_{\psi}} K_{j} d x \geq \int_{U_{\psi}} K_{0} d x=\infty .
$$

Hence

$$
\sup \left\{\int_{U_{\psi}} h d x: h \in \mathcal{H}_{b}\left(U_{\psi}\right), h\left(x_{0}\right)=1\right\}=\infty .
$$

This implies that $U_{\psi}$ fails to have $w$ satisfying (1) and $\inf _{U_{\psi}} w>0$ (cf. [3, Corollary 2.3]). The theorem is proved.

\section{Estimate OF THE GRADIENT OF HARMONIC FUNCTIONS}

By the symbol $c$ we denote a positive constant whose value is unimportant and may change from line to line. If necessary, we use $c_{1}, c_{2}, \ldots$, to specify them. We shall say that two positive functions $f_{1}$ and $f_{2}$ are comparable, written $f_{1} \approx f_{2}$, if and only if there exists a constant $c$ such that $c^{-1} f_{2} \leq f_{1} \leq c f_{2}$.

In this section we shall give a certain lower estimate of the gradient of a positive harmonic function. This estimate will be used for the proof of Theorem (ii). The estimate is based on the following general lemma of A. Ancona, about which Professor Hansen kindly informed the author. The present form of the lemma and its proof are due to Professor Hansen. 
Lemma 1. Let $0<c_{1}<1,0<c_{2}<1$ and $0<A<1$. Then there exists a, $0<$ $a<1$, depending only on $c_{1}, c_{2}$ and $A$ such that for any positive harmonic function $u$ on $B(0,1)$ with $u(0)=1$ and $\inf u\left(B\left(0, c_{2}\right)\right) \leq 1-A$, we have $\inf u\left(B\left(0, c_{1}\right)\right) \leq$ $1-a$.

Proof. It suffices to consider the case $0<c_{1}<c_{2}<1$. Suppose the contrary. Then there exists a sequence $\left\{u_{j}\right\}$ of positive harmonic functions on $B(0,1)$ such that $u_{j}(0)=1, \inf u_{j}\left(B\left(0, c_{2}\right)\right) \leq 1-A$ and $\lim _{j \rightarrow \infty}\left(\inf u_{j}\left(B\left(0, c_{1}\right)\right)\right)=1$. Taking a locally convergent subsequence, we obtain a positive harmonic function $u$ on $B(0,1)$ such that $\inf u\left(B\left(0, c_{2}\right)\right) \leq 1-A$ and $u(0)=1=\inf u\left(B\left(0, c_{1}\right)\right)$. By the minimum principle $u=1$ on $B\left(0, c_{1}\right)$ and hence on $B(0,1)$ by analyticity. Thus a contradiction arises.

By translation and dilation we obtain the following corollary.

Corollary. Let $0<c_{1}<1,0<c_{2}<1$ and $0<A<1$ and let $0<a<1$ be as in Lemma 1. Let $x \in \mathbb{R}^{n}$ and $r>0$. Then for any positive harmonic function $u$ on $B(x, r)$ with inf $u\left(B\left(x, c_{2} r\right)\right) \leq(1-A) u(x)$, we have inf $u\left(B\left(x, c_{1} r\right)\right) \leq(1-a) u(x)$.

From this corollary we obtain the following lower estimate of the gradient of a positive harmonic function, which may be of some independent interest. By $m(E)$ we denote the Lebesgue measure of $E$.

Lemma 2 (cf. [1, Lemma 1]). Let $0<c_{1}<1,0<c_{2}<1$ and $0<A<1$. Then there exist positive constants $\varepsilon$ and $c_{3}$ depending only on $c_{1}, c_{2}$ and $A$ with the following property: Let $x \in \mathbb{R}^{n}$ and $r>0$. If $u$ is a positive harmonic function on $B(x, r)$ and there is $x^{*} \in B\left(x, c_{2} r\right)$ such that $u\left(x^{*}\right) \leq(1-A) u(x)$, then

$$
m\left(\left\{y \in B\left(x, c_{1} r\right):|\nabla u(y)| \geq \varepsilon \frac{u(x)}{r}\right\}\right) \geq c_{3} r^{n} .
$$

Moreover, for $1 \leq p<\infty$, there exists a positive constant $c_{p}$ depending only on $c_{1}$, $c_{2}, A$ and $p$ such that

$$
u(x)^{p} \leq c_{p} r^{p-n} \int_{B\left(x, c_{1} r\right)}|\nabla u|^{p} d y .
$$

Proof. Let $a$ be as in Lemma 1. Then, by Corollary, we find $x^{\prime} \in \bar{B}\left(x, c_{1} r\right)$ such that $u\left(x^{\prime}\right) \leq(1-a) u(x)$. By the Harnack inequality, we find $c_{4}, 0<c_{4}<c_{1}$, depending only on $a$ such that $u(y) \geq(1-a / 2) u(x)$ for $y \in B\left(x, c_{4} r\right)$. Hence

$$
u(y)-u\left(x^{\prime}\right) \geq \frac{a}{2} u(x) \quad \text { for } y \in B\left(x, c_{4} r\right) .
$$

Let $\ell_{y}$ be the line segment connecting $y$ and $x^{\prime}$ for $y \in B\left(x, c_{4} r\right)$. We observe that $\ell_{y} \subset \bar{B}\left(x, c_{1} r\right)$ and so the the length $\left|\ell_{y}\right|$ of $\ell_{y}$ is uniformly bounded by $2 c_{1} r$. On the other hand, representing $u$ as the Poisson integral and differentiating under the integral sign, we obtain

$$
|\nabla u(z)| \leq c_{5} \frac{u(x)}{r} \quad \text { for } z \in B\left(x, c_{1} r\right) .
$$


Now let $\varepsilon=a /\left(8 c_{1}\right)$. We have from (2) and (3)

$$
\begin{aligned}
\frac{a}{2} u(x) & \leq u(y)-u\left(x^{\prime}\right) \leq \int_{\ell_{y}}|\nabla u| d s \\
& \leq \int_{\left\{z \in \ell_{y}:|\nabla u(z)|<\varepsilon u(x) / r\right\}} \varepsilon \frac{u(x)}{r} d s+\int_{\left\{z \in \ell_{y}:|\nabla u(z)| \geq \varepsilon u(x) / r\right\}} c_{5} \frac{u(x)}{r} d s \\
& \leq 2 c_{1} r \varepsilon \frac{u(x)}{r}+c_{5} \frac{u(x)}{r}\left|\left\{z \in \ell_{y}:|\nabla u(z)| \geq \varepsilon \frac{u(x)}{r}\right\}\right| \\
& =\frac{a}{4} u(x)+c_{5} \frac{u(x)}{r}\left|\left\{z \in \ell_{y}:|\nabla u(z)| \geq \varepsilon \frac{u(x)}{r}\right\}\right|
\end{aligned}
$$

uniformly for $y \in B\left(x, c_{4} r\right)$. Hence

$$
\left|\left\{z \in \ell_{y}:|\nabla u(z)| \geq \varepsilon \frac{u(x)}{r}\right\}\right| \geq \frac{a}{4 c_{5}} r .
$$

Since the above inequality holds uniformly for $y \in B\left(x, c_{4} r\right)$, Fubini's theorem yields the required estimate.

Now we integrate $|\nabla u|$ over $B\left(x, c_{1} r\right)$. We have

$$
\int_{B\left(x, c_{1} r\right)}|\nabla u| d y \geq \varepsilon \frac{u(x)}{r} c_{3} r^{n},
$$

whence, by Hölder's inequality, for $1 \leq p<\infty$,

$$
\frac{u(x)}{r} \leq c \frac{1}{m\left(B\left(x, c_{1} r\right)\right)} \int_{B\left(x, c_{1} r\right)}|\nabla u| d y \leq c\left(\frac{1}{m\left(B\left(x, c_{1} r\right)\right)} \int_{B\left(x, c_{1} r\right)}|\nabla u|^{p} d y\right)^{1 / p} .
$$

This readily yields the second assertion.

\section{Proof of Theorem (ii)}

Hereafter, we let $D$ be a $k$-Lipschitz domain with $0<k<1 / \sqrt{n-1}$. By $\delta(x)$ we denote the distance $\operatorname{dist}(x, \complement D)$. Let us recall that $x_{0} \in D$ is a fixed point and put $g(x)=G\left(x, x_{0}\right)$, where $G$ is the Green function for $D$. The function $g$ is positive and harmonic in $D \backslash\left\{x_{0}\right\}$ and vanishes on $\partial D$. Let $r_{0}=\frac{1}{3} \delta\left(x_{0}\right)$. It is easy to see that $x_{0} \notin B\left(\xi, 3 r_{0}\right)$ for any $\xi \in \partial D$.

Lemma 3. There exists a constant $c_{6}, 0<c_{6}<1$, depending only on $D$ such that if $x \in D$ and $\delta(x)<r_{0}$, then there is $x^{*} \in B\left(x, c_{6} \delta(x)\right)$ such that $g\left(x^{*}\right) \leq \frac{1}{2} g(x)$.

Proof. Let $x \in D$ and $\delta(x)<r_{0}$. Take $\bar{x} \in \partial D$ such that $|x-\bar{x}|=\delta(x)$. We observe that $g$ is a positive harmonic function on $D \cap B(\bar{x}, 3 \delta(x))$ which vanishes on $\partial D$. The boundary Harnack principle (e.g. [4, Lemma 4.10]) yields

$$
g \approx g(x) \omega(\cdot, D \cap \partial B(\bar{x}, 2 \delta(x))) \quad \text { on } D \cap B(\bar{x}, \delta(x)),
$$

where $\omega$ denotes the harmonic measure. A Lipschitz domain is uniformly $\Delta$-regular (cf. [2]) and there is $\kappa>0$ depending only on $D$ such that for $0<\rho<2$

$$
\omega(\cdot, D \cap \partial B(\bar{x}, 2 \delta(x))) \leq c \rho^{\kappa} \quad \text { on } D \cap B(\bar{x}, \rho \delta(x)) .
$$

Hence, if $\rho$ is sufficiently small, then

$$
g \leq \frac{1}{2} g(x) \quad \text { on } D \cap B(\bar{x}, \rho \delta(x)) ;
$$


in particular, we find $x^{*} \in B(x,(1-\rho / 2) \delta(x))$ such that $g\left(x^{*}\right) \leq \frac{1}{2} g(x)$. Thus the lemma follows with $c_{6}=1-\rho / 2$.

For $x \in D$ we let

$$
B^{*}(x)=\left\{y \in D:|x-y|<\frac{1}{2} \delta(y)\right\} .
$$

Then, it is easy to see that

$$
B\left(x, \frac{1}{3} \delta(x)\right) \subset B^{*}(x) .
$$

In fact, if $y \in B\left(x, \frac{1}{3} \delta(x)\right)$, then $|x-y|<\frac{1}{3} \delta(x)$ and $\frac{2}{3} \delta(x)<\delta(y)<\frac{4}{3} \delta(x)$, whence $|x-y|<\frac{1}{2} \delta(y)$. With the aid of Lemma 3, we can invoke Lemma 2 with $c_{1}=\frac{1}{3}$, $c_{2}=c_{6}, A=\frac{1}{2}, r=\delta(x), u=g$ and $p=2$. We have

$$
\int_{B^{*}(x)}|\nabla g|^{2} d y \geq \int_{B\left(x, \frac{1}{3} \delta(x)\right)}|\nabla g|^{2} d y \geq c \delta(x)^{n-2} g(x)^{2}
$$

for $x \in D$ with $\delta(x)<r_{0}$. Now we are ready to prove Theorem (ii).

Proof of Theorem (ii). We find $\gamma>0$ such that

$$
\{x \in D: g(x)<\gamma\} \subset\left\{x \in D: \delta(x)<r_{0}\right\} .
$$

As was observed in Section 2, the function $u_{\psi}$ is homogeneous of degree 2 for $\cos \psi=1 / \sqrt{n}$. By the comparison of $g$ and $u_{\psi}$, we can find $\alpha, 1<\alpha<2$, such that

$$
g(x) \geq c \delta(x)^{\alpha} \quad \text { for } x \in D,
$$

since the domain $D$ is $k$-Lipschitz with $0<k<1 / \sqrt{n-1}$. Let $\eta=(2-\alpha) / \alpha$ and define

$$
w^{*}(x)= \begin{cases}|\nabla g(x)|^{2} g(x)^{-2} & \text { if } g(x) \geq \gamma, \\ |\nabla g(x)|^{2} g(x)^{\eta-1} & \text { if } g(x)<\gamma .\end{cases}
$$

Let $h \in \mathcal{H}_{b}$. In the same way as in [1, Section 2], we have from the coarea formula and the Poisson integral formula

$$
\begin{aligned}
\int_{D} h w^{*} d x & =\int_{0}^{\gamma} d t \int_{\{g=t\}} h|\nabla g|^{2} g^{\eta-1} \frac{d \sigma}{|\nabla g|}+\int_{\gamma}^{\infty} d t \int_{\{g=t\}} h|\nabla g|^{2} g^{-2} \frac{d \sigma}{|\nabla g|} \\
& =\int_{0}^{\gamma} t^{\eta-1} d t \int_{\{g=t\}} h|\nabla g| d \sigma+\int_{\gamma}^{\infty} t^{-2} d t \int_{\{g=t\}} h|\nabla g| d \sigma \\
& =h\left(x_{0}\right)\left(\int_{0}^{\gamma} t^{\eta-1} d t+\int_{\gamma}^{\infty} t^{-2} d t\right)=\left(\frac{\gamma^{\eta}}{\eta}+\frac{1}{\gamma}\right) h\left(x_{0}\right) .
\end{aligned}
$$

Next we mollify $w^{*}$. Fix a radially symmetric smooth function $\tau \geq 0$ on $\mathbb{R}^{n}$ such that $\{\tau>0\}=B(0,1)$ and $\int_{\mathbb{R}^{n}} \tau d x=1$. For $y \in D$ we define

$$
\tau_{y}: x \mapsto \frac{1}{\left(\frac{2}{3} \delta(y)\right)^{n}} \tau\left(\frac{x-y}{\frac{2}{3} \delta(y)}\right), \quad x \in \mathbb{R}^{n} .
$$

Let

$$
w(x)=\int_{D} \tau_{y}(x) w^{*}(y) d y
$$


It is easy to see that $w$ is a positive smooth function on $D$. By Fubini's theorem, (6) and the mean value property of $h \in \mathcal{H}_{b}$, we have

$$
\begin{aligned}
\int_{D} h(x) w(x) d x & =\int_{D} h(x) d x \int_{D} \tau_{y}(x) w^{*}(y) d y \\
& =\int_{D} w^{*}(y) d y \int_{D} h(x) \tau_{y}(x) d x \\
& =\int_{D} w^{*}(y) h(y) d y=\left(\frac{\gamma^{\eta}}{\eta}+\frac{1}{\gamma}\right) h\left(x_{0}\right) .
\end{aligned}
$$

Thus $w$ satisfies (1). Let

$$
V=\left\{x \in D: g<\gamma \text { on } B^{*}(x)\right\} .
$$

We observe that $D \backslash V$ is a compact subset of $D$ and $w \geq c$ on $D \backslash V$. Hence we have only to show

$$
w \geq c \quad \text { on } V .
$$

We observe that

$$
\tau_{y}(x)=\frac{1}{\left(\frac{2}{3} \delta(y)\right)^{n}} \tau\left(\frac{x-y}{\frac{2}{3} \delta(y)}\right) \geq \frac{\inf \tau\left(B\left(0, \frac{3}{4}\right)\right)}{\left(\frac{2}{3} \delta(y)\right)^{n}} \geq c \delta(x)^{-n}
$$

for $y \in B^{*}(x)=\left\{y:|x-y|<\frac{1}{2} \delta(y)\right\}$. Suppose $x \in V$. Then $w^{*}=|\nabla g|^{2} g^{\eta-1} \approx$ $|\nabla g|^{2} g(x)^{\eta-1}$ on $B^{*}(x)$ by the Harnack inequality. It follows from (4) that

$$
\begin{aligned}
w(x) & \geq \int_{B^{*}(x)} \tau_{y}(x) w^{*}(y) d y \geq c \delta(x)^{-n} g(x)^{\eta-1} \int_{B^{*}(x)}|\nabla g|^{2} d y \\
& \geq c \delta(x)^{-n} g(x)^{\eta-1} \delta(x)^{n-2} g(x)^{2}=c g(x)^{\eta+1} \delta(x)^{-2} .
\end{aligned}
$$

By (5) and the definition of $\eta$ we have

$$
w(x) \geq c g(x)^{\eta+1} \delta(x)^{-2} \geq c \delta(x)^{\alpha(\eta+1)-2}=c .
$$

The theorem follows.

\section{REFERENCES}

1. H. Aikawa, Integrability of superharmonic functions and subharmonic functions, Proc. Amer. Math. Soc. 120 (1994), 109-117. MR 94b:31003

2. A. Ancona, On strong barriers and an inequality of Hardy for domains in $\mathbb{R}^{n}$, J. London Math. Soc. (2) 34 (1986), 274-290. MR 87k:31004

3. W. Hansen and I. Netuka, Volume densities with the mean value property for harmonic functions, Proc. Amer. Math. Soc. 123 (1995), 135-140. MR 95c:31002

4. D. S. Jerison and C. E. Kenig, Boundary behavior of harmonic functions in non-tangentially accessible domains, Adv. in Math. 46 (1982), 80-147. MR 84d:31005b

5. K.-O. Widman, Inequalities for the Green function and boundary continuity of the gradient of solutions of elliptic differential equations, Math. Scand. 21 (1967), 17-37. MR 39:621

Department of Mathematics, Shimane University, Matsue 690, Japan

E-mail address: haikawa@riko.shimane-u.ac.jp 